\title{
Von militärischer Notwendigkeit zu individueller Verantwort- lichkeit - Der Fall „AI Mahdi“ als Zäsur in der strafrechtlichen Ahndung von Kulturgutzerstörung auf internationaler Ebene
}

\author{
Maximilian Gröber \\ Kerngebiet: Zeitgeschichte \\ eingereicht bei: assoz. Prof. Mag. Dr. Eva Pfanzelter, MA \\ eingereicht im: WiSe 2018/19 \\ Rubrik: Seminar-Arbeit
}

\begin{abstract}
From military necessity to individual responsibility - the "Al Mahdi" case as a caesura in the criminal punishment of the destruction of cultural property on an international level

In April 2012, the Islamist group Ansar Dine launched an attack on the UNESCO World Heritage sites in Timbuktu, Mali. Besides illustrating the political and religious backgrounds that led to this action, the purpose of this paper is to highlight the histori-cal development of the international legal framework for the protection of cultural her-itage, including the trial against Ahmad Al Faqi Al Mahdi, who was in charge of the Timbuktu attacks. As will be shown, the case has the potential to set a precedent.
\end{abstract}

\section{Einleitung}

"Let us be clear: what is at stake here is not just walls and stones. The destroyed mausoleums were important, from a religious point of view, from an historical point of view, and from an identity point of view. Such an attack against buildings dedicated to religion and historic monuments falls into the category of crimes that destroy the roots of an entire people."

International Criminal Court (Hrsg.), Statement of the Prosecutor of the International Criminal Court, Fatou Bensouda, at the opening of the confirmation of charges hearing in the case against Mr Ahmad Al-Faqi Al Mahdi, 
Der vor dem Internationalen Strafgerichtshof (IStGH) geführte Prozess gegen Ahmad Al Faqi Al Mahdi, der 2012 als ideologischer Rädelsführer einer islamistischen Terrorgruppe maßgeblich für die Zerstörung der in Timbuktu, Mali, angesiedelten Welterbestätten verantwortlich war, stellte nur den Gipfel einer jahrzehntelangen Entwicklung dar. Während die weltweite Staatengemeinschaft unter dem Eindruck zweier Weltkriege zunächst versuchte, ihre Militärs durch international festgelegte Richtlinien im Umgang mit Kulturgütern zu sensibilisieren, bahnte sich mit der Bildung von Terrororganisationen im ausgehenden 20. Jahrhundert eine neue Form der Bedrohung für das kulturelle Erbe der Menschheit an. Beide Aspekte, also sowohl die historische Entwicklung von überstaatlichen Abkommen zugunsten des Kulturgüterschutzes als auch die Hintergründe der modernen Bedrohungslage durch radikale, bewaffnete Gruppierungen, sollen in dieser Arbeit beleuchtet werden. Illustriert am Verfahren gegen Ahmad Al Mahdi steht die Frage im Zentrum, welche rechtlichen Mittel der internationalen Gemeinschaft und ihren Institutionen bzw. Organisationen gegen die absichtliche Zerstörung von Kulturgütern durch nichtstaatliche Akteur*innen zur Verfügung stehen. Dabei wird von der These ausgegangen, dass das Urteil gegen Al Mahdi eine Zäsur in der strafrechtlichen Ahnung der Zerstörung von Kulturgütern auf internationaler Ebene darstellt.

Um den Tathergang, der später zum Prozess führte, nachvollziehen zu können, soll zunächst auf die Entstehung und Bedeutung der Kulturstätten Timbuktus sowie die politischen Umwälzungen seit der Unabhängigkeit Malis 1960 eingegangen werden. Der daraus erwachsene ethnisch-kulturelle Konflikt zwischen den nomadisierenden Tuareg-Stämmen im Norden des Landes und der Staatsgewalt bildete den Nährboden für die Entstehung militanter Gruppen in der Region, die später den Angriff auf die Mausoleen Timbuktus leiteten. Parallel zur Beschreibung dieser Ereignisse werden im dritten Abschnitt der Arbeit die seit dem 19. Jahrhundert aufkommenden Versuche zum rechtlich verankerten Schutz von Kulturgütern skizziert.

Bedingt durch die Aktualität des Themas konnte zur Bearbeitung der oben dargelegten Fragestellungen nicht im gewohnten Umfang auf historische Fachliteratur zurückgegriffen werden. Unter Anbetracht dieser Tatsache bildet die umfangreiche Monografie Sabine von Schorlemers eine wertvolle Stütze der Arbeit. ${ }^{2}$ Von Schorlemer charakterisiert darin nicht nur die Motive von Zerstörungsakten gegen Kulturstätten im 21. Jahrhundert, sondern stellt auch stets den Bezug zur zeitgenössischen internationalen Rechtsprechung und ihren Grundlagen her. Was den historischen Kulturgüterschutz betrifft, sei der Aufsatz von Berenika Drazewska erwähnt, ${ }^{3}$ der eine kompakte Übersicht zu den wichtigsten Dokumenten und ihrer Weiterentwicklung bietet. Ein auch für Nicht-Jurist*innen gut verständliches Werk, welches das Völkerstrafrecht - in

1.3.2016, [https://www.icc-cpi.int/Pages/item.aspx?name=otp-stat-01-03-16], eingesehen 20.2.2019.

2 Sabine von Schorlemer, Kulturgutzerstörung. Die Auslöschung von Kulturerbe in Krisenländern als Herausforderung für die Vereinten Nationen (The United Nations and Global Change 11), Baden-Baden 2016.

3 Berenika Drazewska, The Human Dimension of the Protection of the Cultural Heritage from Destruction during Armed Conflicts, in: International Journal of Cultural Property 22 (2015), Heft 2-3, S. 205-228. 
erster Linie rechtlich, aber ebenso historisch - behandelt, stammt von Gerhard Werle. ${ }^{4}$ Es bildet das Fundament des rechtlichen Teils der Arbeit, der im dritten Abschnitt dargelegt wird. Für die abschließende juristische Bewertung des Falles Al Mahdi wurden vor allem aktuelle Aufsätze aus rechtswissenschaftlichen Fachzeitschriften verwendet. Abseits der großen (rechts-)historischen Entwicklungslinien erfolgte die Rekonstruktion der Geschehnisse in Mali und des darauffolgenden Prozesses fast gänzlich anhand von Artikeln verschiedener Zeitungen, die zumeist online erschienen, sowie durch die Beiträge aus dem Webauftritt der UNESCO und den für die Öffentlichkeit anschaulich aufbereiteten Unterlagen des IStGH. ${ }^{5}$

Um eine einheitliche Schreibweise zu gewährleisten, wurde bei den die Kulturstätten Timbuktus betreffenden Eigennamen nach der Schreibart des Informationsmaterials des Internationalen Strafgerichtshofes zitiert. ${ }^{6}$

\section{Mali \& Timbuktu}

\subsection{Die Stadt Timbuktu und ihre Kulturgüter - Ursprung und Bedeutung}

Geografisch befindet sich Timbuktu im Norden Malis respektive am Südrand der Sahara sowie am nördlichen Scheitelpunkt des Flusses Niger. Dies ist insofern relevant, da sich der kulturelle Reichtum der Siedlung erst durch ihre geografische Lage, die sie zu einem bedeutenden Verkehrsknotenpunkt im Transsaharahandel machte, nachvollziehen lässt. Die mit den Karawanen eintreffenden Waren konnten von Timbuktu aus eingeschifft und weitertransportiert werden. ${ }^{7}$ Während die Stadt selbst bereits im 5. Jahrhundert gegründet wurde, lag ihre Blütezeit im 15. und 16. Jahrhundert. ${ }^{8}$ Zusammen mit anderen Orten der Region wie Djenné oder Gao belegen die Siedlungsstrukturen der Sahel-Sudan-Zone insgesamt eine hoch entwickelte präkoloniale Stadtkultur, welche mediterrane, nordafrikanische und islamische Strukturelemente beinhaltete, denn neben der Bedeutung als Handelszentrum diente Timbuktu seit dem 12. Jahrhundert als Ausgangsbasis für die Verbreitung des Islams im Westsudan. ${ }^{9}$ Die ansässige Universität von Sankore sowie zahlreiche Koranschulen sollen dabei bis zu 25.000 Studierenden die islamische Religion und Kultur vermittelt haben. Des Weiteren bezeugen drei große Moscheen die damalige Bedeutung Timbuktus. Sultan Kankan Moussa erbaute 1325, nachdem er von einer Pilgerreise nach Mekka zurückkehrte, die DjingareyberMoschee, die zwischen 1570 und 1583 restauriert und erweitert wurde. Ihr zentrales Minarett dominiert das Stadtbild bis heute und stellt ein von weitem sichtbares Wahrzeichen dar. In einem ähnlichen Zeitraum, zwischen 1578 und 1582, entstand die

Gerhard Werle, Völkerstrafrecht, Tübingen 2012².

International Criminal Court (Hrsg.), Case Information Sheet. The Prosecutor v. Ahmad Al Faqi Al Mahdi, 20.3.2018, [https://www.icc-cpi.int/Caselnformation Sheets/Al-MahdiEng.pdf], eingesehen 5.1.2019.

Ebd.

Thomas Krings, Sahelländer. Mauretanien, Senegal, Gambia, Mali, Burkina Faso, Niger, Darmstadt 2006, S. 85.

United Nations Educational, Scientific and Cultural Organization (Hrsg.), Timbuktu, o. D., [https://whc.une sco.org/ en/list/119/, eingesehen 5.1.2019.

9 Krings, Sahelländer, S. 83-85. 
Moschee von Sankore. Südlich von ihr befindet sich die Sidi-Yahia-Moschee, die sich auf die Zeit um 1400 zurückdatieren lässt. ${ }^{10}$

Außer den Gotteshäusern umfasste das ursprüngliche UNESCO-Weltkulturerbe von Timbuktu vor den Zerstörungen 2012 noch 16 Grabstätten und Mausoleen, die in der Geschichte des religiösen Brauchtums eine essentielle Rolle einnahmen. Bei ihnen handelte es sich um eine Art von Heiligengräbern, wobei die kleiner dimensionierten und schlicht gestalteten Mausoleen als Marabuts bezeichnet werden. Sie dienten als Grablegen für Gelehrte des Sufismus, einer mystischen Strömung innerhalb des Islams, die sich in allen maghrebinischen und westafrikanischen Ländern über die Jahrhunderte verbreitete, und besaßen einen festen Platz in der Volksfrömmigkeit. Im Verständnis der gläubigen Personen geht von ihnen eine segensvolle Kraft (Baraka) aus. Männer sowie Frauen beten daher aus verschiedensten Anlässen an den Stätten. ${ }^{11}$ Wie sich auch in ihrer Architektur zeigt, stellten die Mausoleen in Timbuktu eine Art Schutzwall dar, welcher die Stadt vor allem Unheil behüten sollte. Das älteste Mausoleum stammt aus dem Jahr 1529 und liegt 150 Meter westlich der Innenstadt. Es beherbergte den Leichnam von Scheich Abdul Kassim Attoutaty sowie von fünfzig Ulemas ${ }^{12}$ und anderen als heilig erachteten Personen. Die in den Mausoleen begrabenen Scheichs leiteten oft Sufi-Bruderschaften, die bis in die Gegenwart das soziale und gesellschaftliche Leben in der Region beeinflussen. ${ }^{13}$ In einem weiteren Bauwerk dieser Epoche wurde der Restaurator der Djingareyber-Moschee, Imam Qadi Al Aqib, beigesetzt. ${ }^{14}$

Nach einer Begutachtung durch die International Council on Monuments and Sites (ICOMOS) 1987 wurde die Altstadt von Timbuktu auf Basis der Selektionskriterien (ii), (iv) und (v) für die UNESCO-Welterbeliste nominiert. ${ }^{15} \mathrm{Im}$ Allgemeinen unterscheidet die UNESCO innerhalb des Terminus der "Welterbestätten“ zwischen einem „Weltkulturerbe“ und einem „Weltnaturerbe“, das anhand von zehn Kriterien ausgewählt werden kann, wobei mindestens eines davon erfüllt sein muss, um den Status eines Welterbes zu erlangen. Die drei für die Kulturstätten Timbuktus attestierten Kriterien definieren sich wie folgt:

"(ii) to exhibit an important interchange of human values, over a span of time or within a cultural area of the world, on developments in architecture or technology, monumental arts, town-planning or landscape design; [...]

10 International Council on Monuments and Sites (Hrsg.), Advisory Body Evaluation, The Old Town of Timbuktu, No. 119 Rev., 22.12.1987, S. 2, [https://whc.unesco.org/document/152016], eingesehen 5.1.2019.

11 Wolfgang Günter Lerch, Zerstörungen in Mali. Steinzeit-Islam, in: Frankfurter Allgemeine Zeitung, 2.7.2012, [https://www.faz.net/aktuell/politik/ausland/zerstoerungen-in-mali-steinzeit-islam-1 1807372.html], eingesehen 23.2.2019.

12 Als Ulema (auch: Ulamā) wird ein religiöser Gelehrter in islamischen Gemeinschaften bezeichnet. Vor allem zur Zeit des frühen Islams waren die Ulemas eine einflussreiche Gesellschaftsschicht, die über theologische und juristische Problemstellungen entschieden und ihre Gemeinden somit über Generationen prägen konnten: Asma Afsaruddin, ulamā, in: Encyclopædia Britannica, 27.3.2018, [https://www.britannica.com/topic/ulama], eingesehen 16.2.2019.

13 Lerch, Zerstörungen in Mali.

14 International Council on Monuments and Sites, Advisory Body Evaluation, S. 2.

15 Ebd., S. 3. 
(iv)tobeanoutstanding exampleofatypeofbuilding,architecturalortechnological ensemble or landscape which illustrates (a) significant stage(s) in human history; (v) to be an outstanding example of a traditional human settlement, land-use, or sea-use which is representative of a culture (or cultures), or human interaction with the environment especially when it has become vulnerable under the impact of irreversible change ${ }^{\prime \prime 16}$

Im Bericht der ICOMOS trifft dabei das Kriterium (ii) zu, da die Moscheen und heiligen Stätten von Timbuktu eine essentielle Rolle bei der frühen Verbreitung des Islam in Afrika spielten. Das vierte Selektionsmerkmal legitimiert sich durch die drei großen Moscheen, in denen sich zum Zeitpunkt ihrer Restauration im 16. Jahrhundert die Bedeutung der Stadt als intellektuelles und spirituelles Zentrum widerspiegelte. Das letztgenannte, fünfte Kriterium nimmt auf die traditionelle Bauweise der Mausoleen und Moscheen Bezug, welche einen repräsentativen Charakter besitzen. ${ }^{17}$ Dieser im Binnendelta des Niger verbreitete städtische Lehmbaustil (oder auch „sudanische Baustil") entwickelte sich über Jahrhunderte in Handelsstädten wie Djenné oder Timbuktu. Er wurde sowohl von animistischen Völkern im Süden des heutigen Malis sowie später von islamisch-nordafrikanischen Strukturelementen beeinflusst. ${ }^{18}$ Im Kontrast zu Bauweisen auf dem Lande unterscheidet sich die städtische Architektur durch Auffälligkeiten in ihrer Dimension sowie in der dekorativen Gestaltung der Fassaden. Der aus dem großen Sedimentationsbecken des Niger gewonnene Lehm war reichlich verfügbar und diente somit als günstiges Baumaterial. Nach der Vermengung mit Wasser, Stroh und Dung konnte der Lehm entweder händisch oder mittels Holzformen zu Ziegeln modelliert werden, die anschließend durch die Sonne getrocknet wurden. ${ }^{19}$

Vor den Attacken 2012 bestand eine Bedrohung der Kulturstätten in der Veränderung ihrer Umgebung. Durch die rasante Urbanisierung der Region wurde die traditionelle Architektur, wobei auch Ensembles wie größere Plätze und Märkte betroffen waren, gefährdet. Die Errichtung moderner Bauten führte unweigerlich zum Bruch mit dem charakteristischen Siedlungsbild. Neben den allgemein zu geringen Wartungs- und Restaurationsmaßnahmen, die vor allem die weniger beachteten Mausoleen in Mitleidenschaft zogen, erwiesen sich starke Regenfälle und die damit einhergehenden Überflutungen als großes Problem. Daher befand sich Timbuktu von 1990 bis 2005 auf der "Roten Liste“ des gefährdeten Erbes der Welt. ${ }^{20}$ Seit 2005 präsentierte die UNESCO mehrere Maßnahmenkataloge, welche die Sanierung und Erhaltung der Kulturgüter,

16 United Nations Educational, Scientific and Cultural Organization (Hrsg.), The Criteria for Selection, O. D., [https:// whc.unesco.org/en/criteria/], eingesehen 16.2.2019.

17 International Council on Monuments and Sites, Advisory Body Evaluation, S. 3

18 Thomas Krings, Die Tradition der urbanen Lehmarchitektur im Obernigergebiet von Mali. Kulturgeographische Beobachtungen zum „sudanischen Baustil“ in der Stadt Djenné (Mali), in: Die Erde 115 (1984), Heft 1, S. 123-144, hier S. 123.

19 Ebd., S. 126

20 Welche Welterbestätten als gefährdet gelten, entscheiden die in dem 1972 verabschiedeten „Übereinkommen zum Schutz des Kultur- und Naturerbes der Menschheit“ (oder auch „Welterbekonvention“) festgelegten Kriterien: Österreichische UNESCO-Kommission (Hrsg.), Die Konvention. Übereinkommen zum Schutz des Kultur- und Naturerbes der Welt, o. D., [https://www.unesco .at/kultur/welterbe/die-konvention/], eingesehen 16.2.2019. 
mit besonderer Berücksichtigung der Altstadt Timbuktus, in Angriff nahmen. ${ }^{21}$ Neben den von der ICOMOS bzw. UNESCO beachteten Welterbestätten befanden sich schätzungsweise noch 700.000 Manuskripte, verteilt auf sechzig private Bibliotheken, ${ }^{22}$ in Timbuktu, die bei den Angriffen 2012 ebenfalls beschädigt wurden. ${ }^{23}$

Zuletzt anzumerken ist, dass die Kulturdenkmäler der Stadt nicht erst mit den Untersuchungen der ICOMOS in den Blick westlicher Forscher*innen gerieten. Bereits der aus Hamburg stammende Geograf, Historiker und Philologe Heinrich Barth verbrachte um die Mitte des 19. Jahrhunderts über ein halbes Jahr in Timbuktu, das er ausführlich in seinen „Reisen und Entdeckungen“ schilderte. Abseits des üblichen kolonialen Überlegenheitshabitus seiner Zeit geht aus Barths Werk eine überaus große Wertschätzung für den west- und zentralafrikanischen Kulturraum hervor. ${ }^{24}$

\subsection{Politische Entwicklung Malis seit 1960}

Wie eine Vielzahl anderer afrikanischer Staaten konnte Mali in der zweiten Hälfte des 20. Jahrhunderts seine Unabhängigkeit von der französischen Kolonialmacht erlangen. Mit Mali entstand 1960 ein ethnisch und kulturell pluraler Staat, in dem insgesamt - wenn die verschiedenen Tuareg-Clans als ein homogenes Kollektiv gewertet werden - elf unterschiedliche Bevölkerungsgruppen leben. ${ }^{25}$ Von Beginn an standen die nomadisierenden Tuareg-Stämme, die etwa zwei Prozent der Population des westafrikanischen Binnenstaats ausmachen, ${ }^{26}$ in einem besonderen Spannungsverhältnis zur Regierung. Bereits 1962 kam es zu einem ersten Aufstand derTuareg gegen die Zentralgewalt, welche versuchte, in ihre nomadische Lebensweise einzugreifen. Die damalige Regierung unter Präsident Modibo Keita hatte nach dem Abrücken Frankreichs die Viehbestände mit hohen Steuern belegt, was in den nördlichen Regionen als Affront empfunden wurde. Hintergründig schwelte außerdem ein ethnischer Konflikt zwischen den sesshaften, afrikanischen Bevölkerungsteilen und den Tuareg-Clans, die in sich geschlossene, streng hierarchisierte Gesellschaften darstellten. Zu ihrer von der politischen Führung des Landes angestrebten Assimilation war es daher kaum gekommen. Der in der Umgebung von Timbuktu und Gao ausgehende Aufstand der Tuareg konnte vom dem malischen Militär mit brutalsten Mitteln innerhalb von zwei Jahren niedergeschlagen werden und endete 1964. Als Folge kam es zur Flucht vieler Tuareg nach Algerien sowie zu öffentlichen Hinrichtungen der Rebellenführer*innen. ${ }^{27}$ Während der folgenden Jahre verfolgte Keita mit der Verstaatlichung von Produktionsmitteln eine sozialistische Agenda, die bei den Nomadenstämmen ebenfalls auf wenig Verständnis stieß. Seine

21 United Nations Educational, Scientific and Cultural Organization, Timbuktu.

22 Timbuktu shrines damaged by Mali Ansar Dine Islamists, in: BBC News, 30.6.2012, [https://www. bbc.com/news/ world-africa-18657463], eingesehen 19.2.2019.

23 Schorlemer, Kulturgutzerstörung, S. 662.

24 Rainer Tetzlaff, Afrika. Eine Einführung in Geschichte, Politik und Gesellschaft, Wiesbaden 2018, S. 74.

25 Laut den von Tetzlaff angegebenen Zahlen aus dem Jahr 2016 stellte die ethnische Gruppe der Bambara mit einem Anteil von 37 \% den größten Anteil der Bevölkerung, gefolgt von den Fulbe (14\%), Senufo (9,5\%), Soninke (9\%), Dogon (8\%), Songhai (7\%), Malinke (7\%), Diola (3\%), Bobo, Oule und Tuareg (jeweils 2 \%): ebd., S. 273.

27 Stefan Brüne, Problemregion Mali Nord. Ein entwicklungspolitischer Rückblick, in: Sicherheit und Frieden 32 (2014), Heft 2, S. 86-90, hier S. 87. 
Amtszeit wurde durch einen Putsch von Seiten des Militärs 1968 beendet.28

Unter seinem Nachfolger, Moussa Traoré, der das Land bis 1991 regierte, verbesserte sich die Situation der Tuareg ebenso wenig. Erneut kam es zu Fluchtbewegungen ins Ausland, die dieses Mal aber nicht primär den politischen, sondern vielmehr ökologischen Umständen geschuldet waren. Zwei große Dürreperioden (1973/74 sowie 1983-86) ${ }^{29}$ verursachten einen massiven Niedergang des Viehbestandes, bei dem schätzungsweise vierzig bis fünfzig Prozent der Tiere zugrunde gingen und wodurch erneut Migrationsbewegungen nach Algerien und Libyen ausgelöst wurden. ${ }^{30}$ Der dortige Machthaber Muammar al-Gaddafi verschaffte den Flüchtenden Arbeit auf den libyschen Ölfeldern und arrangierte eine militärische Ausbildung innerhalb seiner "Islamischen Legion".31 Ab 1980 sprach er sich für eine unabhängige Tuareg-Republik aus. $^{32}$

Die weitgehend stagnierende Wirtschaftsleistung sowie die steigende Verschuldung des Landes provozierten Anfang der 1990er-Jahre zivilgesellschaftliche Proteste von Schüler*innen, Studierenden und Gewerkschafter*innen, die schließlich in der Absetzung Traorés am 26. März 1991 durch sein Offizierskorps mündeten. Bereits mit Juli des vorhergehenden Jahres war abermals ein Tuareg-Aufstand entbrannt - diesmal im Zeichen der Forderung nach Autonomie. Durch ein in Tamanrasset (Algerien) unterzeichnetes Waffenstillstandsabkommen, das noch zwei Monate vor dem Sturz von Traoré verhandelt wurde, versuchte die Zentralregierung mit mehreren Maßnahmen die innenpolitische Situation zu entspannen. Neben einem teilweisen Abzug der malischen Armee sollten den drei nördlichen Provinzen des Landes - Timbuktu, Kidal und Gao - ein Autonomiestatus gewährt sowie ein Investitionsprogramm in Aussicht gestellt werden. Nach dem 26. März bestätigte die neue Übergangsregierung diesen Sonderstatus, den sie allerdings nur unklar definierte, forderte im Gegenzug aber die Eingliederung einer gewissen Anzahl von Tuareg-Soldat*innen in die nationalen Streitkräfte. Des Weiteren waren Programme zur Reintegration von geflüchteten Personen geplant. Wegen der mangelhaften Umsetzung des Vereinbarten kam es in den nächsten Jahren weiterhin zu Auseinandersetzungen, die offiziell erst im März 1997 mit einer Versöhnungszeremonie und der feierlichen Auflösung mehrerer Rebellengruppen ihren Abschluss fanden - ein Frieden auf Zeit. ${ }^{33}$

Nach einer relativ gewaltfreien Periode eskalierte die Lage erneut nach den Erhebungen in Libyen 2011 und dem daraus resultierenden Fall Gaddafis. Am Konflikt beteiligte Tuareg-Kämpfer*innen, welche für Gaddafi als Söldner*innen tätig waren, kehrten in ihre Heimatregionen zurück, was ein Wiederaufflammen der alten Spannungen zur

28 Tetzlaff, Afrika, S. 273.

29 Brüne, Problemregion Mali Nord, S. 87.

30 Tetzlaff, Afrika, S. 273-274.

31 Die „Islamische Legion" wurde 1972 von Gaddafi, mit dem erklärten Ziel, einen großen islamischen Staat in der Sahel-Zone errichten zu wollen, gegründet. Ihre Söldner stammten aus dem Sudan, Mali, Tunesien, Ägypten und dem Tschad: Christoph Ehrhardt/Thomas Gutschker, Libyen. Loyal zum Stamm, nicht zum Regime, in: Frankfurter Allgemeine Zeitung, 23.2.2011, [https://www.faz.net/-gq9-y4l8], eingesehen 17.2.2019.

32 Brüne, Problemregion Mali Nord, S. 87.

33 Tetzlaff, Afrika, S. 274. 
Folge hatte. Die Heimkehrer*innen formierten unter der Führung des ehemaligen libyschen Armeeoffiziers und gebürtigen Maliers Mohamed Ag Najem die Mouvement national de libération de l'Azawad (MNLA), die als erste Gruppe öffentlich die Gründung eines komplett unabhängigen Staates Azawad ${ }^{34}$ zum Ziel hatte. ${ }^{35}$ Zur Vertreibung des malischen Militärs aus den nördlichen Landesteilen schloss die MNLA ein Zweckbündnis mit den drei islamistischen Gruppierungen al-Qaïda au Maghreb islamique (AQMI), Mouvement pour I'Unité et le Djihad en Afrique de l'Ouest (MUJAO) sowie Ansar Dine, das allerdings schon bald wieder zerbrechen sollte. Während die Rebellierenden im Norden immer weiter Fuß fassten, kam es zeitgleich zu einem neuerlichen Militärputsch gegen den Staatspräsidenten, dem vorgeworfen wurde, die Lage nicht mehr unter Kontrolle zu haben. Das sich daraus ergebende Machtvakuum förderte allerdings den Erfolg der MNLA und ihrer Verbündeten, wodurch sie im April 2012 den Staat Azawad ausrufen konnten, der allerdings keine internationale Anerkennung fand. ${ }^{36}$

\subsection{Zerstörungen durch islamistische Gruppierungen 2012}

Dass das Bündnis zwischen der MNLA und den islamistischen Terrorgruppen nicht auf Dauer bestehen würde, war angesichts ihrer divergenten Zielsetzungen vorhersehbar. Mit der Proklamation Azawads endete die Zusammenarbeit zwischen den drei Organisationen noch im April 2012. Ideologisch stand die MNLA mit ihrem Ziel, einen säkulären, unabhängigen Staat in Nord-Mali zu gründen, in starkem Kontrast zu den Vorstellungen der Islamist*innen, welche das gesamte Staatsgebiet beanspruchten und die Einführung der Scharia forderten. ${ }^{37}$ Unter dieser versteht ein Großteil der Muslim*innen die "Gesamtheit der von Gott durch den Koran und durch den Propheten Muhammad übermittelten Normen für Glauben, Ethik und Recht des Islam".38 Im Folgenden soll nun vor allem die Rolle von Ansar Dine, als der Organisation, welche im Wesentlichen für die Beschädigung und Zerstörung der kulturellen Stätten Timbuktus verantwortlich gemacht wird, näher beleuchtet werden.

Übersetzt bedeutet Ansar Dine so viel wie „Verteidiger des Glaubens". Die Organisation wurde im Dezember 2011 von lyad Ag Ghali ins Leben gerufen. Zunächst hatte Ghali mit der malischen Regierung als Verhandlungsführer bei Geiselnahmen, die mit AQMI in Verbindung standen, zusammengearbeitet, bevor er in den 1990er-Jahren einen gescheiterten Aufstand der Tuareg anführte. Auch strebte er im Oktober 2011 eine Position an der Spitze der MNLA an, wobei er aufgrund seines Vorhabens, die Scharia einführen zu wollen, abgelehnt wurde. Daraufhin gründete er Ansar Dine. Nach der

34 Als Territorium, welches den Azawad umfassen sollte, werden in der Literatur die drei nordmalischen Provinzen Gao, Kidal und Timbuktu genannt.

35 Thomas Scheen, Al Qaida beteiligt? Tuareg richten Massaker im Norden Malis an, in: Frankfurter Allgemeine Zeitung, 14.2.2012, [https://www.faz.net/-gq5-6xose], eingesehen 18.2.2019.

36 Tetzlaff, Afrika, S. 275.

37 Stanford University (Hrsg.), Ansar Dine. Mapping Militant Organizations, 8.8.2016, [http://web.stanford.edu/ group/mappingmilitants/cgi-bin/groups/view/437\#note25], eingesehen 21.2.2019.

38 Stefan Reichmuth, Scharia, in: Enzyklopädie der Neuzeit Online, o. D., [https://referenceworks.brillonline.com/ entries/enzyklopaedie-der-neuzeit/scharia-a3747000?s.num=0\&s.f.s2_parent=s.f.book.enzyklopaedie-derneuzeit\&s.q=sharia], eingesehen 17.3.2019. 
späteren militärischen Intervention Frankreichs im Januar 2013 tauchte er schließlich unter. ${ }^{39}$

Ideologisch orientierte sich Ansar Dine an der im 18. Jahrhundert von Muhammad Ibn Abdal Wahhab begründeten Lehre des Wahhabismus, welche in den oben erläuterten sufistischen Praktiken eine Überformung und Entartung des Islam sah. Die Verehrung der Mausoleen sowie den bei ihnen bestatteten Heiligen deutete Wahhab als Götzendienst, der bekämpft werden müsse - ein Auftrag, den die Terrororganisation nach dem Bruch mit der MNLA sogleich umzusetzen versuchte. ${ }^{40}$

Nachdem es Ansar Dine im April 2012 gelang, Timbuktu vollständig unter seine Kontrolle zu bringen, begannen am 4. Mai mit einem Brandanschlag auf das Grab des SufiGelehrten Sidi Mahamoud Ben Omar Mohamed Aquit ${ }^{41}$ die ersten Beschädigungen. Ende Juni setzte eine größere Zerstörungswelle ein, welche die Grundlage der späteren Anklage des Internationalen Strafgerichtshofs bildete. ${ }^{42}$ Am 30. Juni 2012 zerstörten Mitglieder der Terrororganisation drei weitere Sufi-Schreine, darunter das komplette Mausoleum von Sidi Mahamoud. Am nächsten Tag erfolgten Angriffe auf die restlichen Mausoleen der Altstadt Timbuktus, die unter anderem mit Spitzhacken und anderen primitiven Werkzeugen, aber auch mit Sturmgewehren vom Typ AK-47 durchgeführt wurden. Dabei kündigte Ansar Dine bereits an, alle Mausoleen ausnahmslos ausradieren zu wollen. ${ }^{43}$

Letztendlich betrafen die Aktionen der Islamist*innen neun Mausoleen sowie eine Moschee, ${ }^{44}$ wobei das Spektrum der Schäden von verhältnismäßig geringen Beschädigungen bis zur kompletten Auslöschung von Mausoleen reichte. Unter den betroffenen Objekten befanden sich neben dem bereits erwähnten Mausoleum von Sidi Mahamoud Ben Omar Mohamed Aquit noch die Mausoleen von Scheich Mohamed Mahmoud Al Arawani, Scheich Sidi Mokhtar Ben Sidi Muhammad Ben Scheich Alkabir, Scheich Sidi Ahmed Ben Amar Arragadi, Scheich Muhammad El Mikki, Scheich Abdoul Kassim Attouaty, die Mausoleen von Alpha Moya, Ahmed Fulane, Bahaber Babadié und die Tür der Sidi-Yahia-Moschee. ${ }^{45}$

Was die Reaktionen der internationalen Gemeinschaft betrifft, so wurde die UNESCO schon vor den konkreten Attacken auf die Bedrohung der Welterbestätten aufmerksam. Bereits kurz nach der Einnahme Timbuktus durch die noch bestehende Rebellenallianz forderte die damalige Generaldirektorin der UNESCO, Irina Bokova, alle Kriegs-

39 Stanford University, Ansar Dine.

40 Lerch, Zerstörungen in Mali.

41 Kristy Campion, Blast through the Past. Terrorist Attacks on Art and Antiquities as a Reconquest of the Modern Jihadi Identity, in: Perspectives on Terrorism 11 (2017), Heft 1, S. 26-39, hier S. 33.

42 Vgl. International Criminal Court, Case Information Sheet.

43 Campion, Blast through the Past, S. 33.

44 Dabei handelte es sich um ein versiegeltes Tor der Sidi-Yahia-Moschee, welches durch Ansar Dine aufgebrochen wurde, womit sich die Angreifer*innen Zugang zu weiteren Heiligengräbern verschaffen konnten. Dies stellte für die ansässige Bevölkerung einen erneuten Schock dar, da die Öffnung des Tores nach den lokalen Glaubensvorstellungen Unheil heraufbeschwören würde: Timbuktu's Sidi Yahia Mosque 'Attacked by Mali Militants', in: BBC News, 2.7.2012, [https://www.bbc.com/news/world-africa-18675539], eingesehen 27.2.2019. 
teilnehmenden zum Schutz der Kulturgüter auf. ${ }^{46}$ Angesichts der sich immer weiter zuspitzenden Lage akzeptierte das Welterbekomitee bei einer Sitzung in St. Petersburg am 28. Juni 2012 - also nur zwei Tage vor den einsetzenden Zerstörungen - die Anfrage der malischen Regierung, Timbuktu auf die „Rote Liste“ des gefährdeten Welterbes zu setzen. ${ }^{47}$ In Anbetracht dieser Tatsachen dürfte davon auszugehen sein, dass der Zeitpunkt des Angriffs auf die Mausoleen nicht zufällig gewählt wurde, sondern eine bewusste Reaktion auf die Handlung des Welterbekomitees respektive auf die durch sie repräsentierte internationale Gemeinschaft darstellte. Die Aussage eines Sprechers von Ansar Dine ("God is unique. All of this is haram [or forbidden in Islam]. We are all Muslims. UNESCO is what?" ${ }^{\prime 48}$ ) legt diesen Verdacht besonders nahe.

Gezielte Angriffe wie dieser passen auch in die Charakterisierung der Ursachen und Motive für Kulturgüterzerstörung im 21. Jahrhundert, die Sabine von Schorlemer in ihrer Monografie darlegt. Trotz - oder gerade wegen - der Bemühungen der Weltgemeinschaft und des von ihr ausgeübten internationalen Drucks kommt es regelmäßig zu Attacken solcher Art, um einen Herrschaftsanspruch nach außen zu demonstrieren. Die vordergründig postulierten religiösen Motive dienen hingegen zur inneren Machtkonsolidierung der Angreifer*innen. Sie zeigen der Zivilbevölkerung, dass neben der Ideologie ihres Regimes kein Spielraum für andere als Häresie empfundene Vorstellungen besteht. ${ }^{49}$ Als berüchtigtes Beispiel wäre hierbei die Vernichtung der BuddhaStatuen von Bamiyan durch Al Qaida im Jahr 2001 zu nennen, welche zum Ziel hatte, die Spuren der buddhistischen Vergangenheit Afghanistans auszulöschen. ${ }^{50}$ In der Literatur ist dabei unter anderem von einem (kulturellen) Ikonoklasmus die Rede. ${ }^{51}$

Zerstörungen, die sich wie jene in Nordmali gegen das eigene kulturelle Erbe der Täter*innen wandten, heben sich hingegen vom Großteil der historischen Zerstörungsakte ab, die gegen Kulturgüter von Feinden gerichtet waren. Schorlemer spricht diesbezüglich von einer subjektiven Fehl-Wahrnehmung von Kultur als etwas „Fremdes" und damit auch Zerstörbares. ${ }^{52}$ Ähnliche Tendenzen zeigte auch der in Syrien tätige sogenannte „Islamische Staat" (IS) mit der Sprengung des Nabi-Junus-Schreins - das im Judentum, Christentum und Islam verehrte Grab des Propheten Jonah und der Vernichtung von sunnitischen theologischen Büchern aus der Bibliothek von Mossul..$^{53}$

46 United Nations Educational, Scientific and Cultural Organization (Hrsg.), UNESCO Director-General Expresses Concern about the Situation in Mali, 2.4.2012, [https://whc.unesco.org/en/news/ 865/], eingesehen 26.2.2019.

47 United Nations Educational, Scientific and Cultural Organization (Hrsg.), Director-General of UNESCO Calls for a Halt to Destruction of Cultural Heritage Site in Timbuktu, 30.6.2012, [https://whc.unesco.org/en/n ews/901/], eingesehen 26.2.2019.

48 Zit. nach Ansar Dine Fighters Destroy Timbuktu Shrines, in: Al Jazeera English, 1.7.2012, [https://www.aljazeera. com/news/africa/2012/06/2012630101748795606.html], eingesehen 26.2.2019.

49 chorlemer, Kulturgutzerstörung, S. 150.

50 Ebd., S.131.

51 Der Begriff "Ikonoklasmus" meint dabei die Ablehnung und Zerstörung kultischer Bilder: Uta Wiggermann, Ikonoklasmus, in: Enzyklopädie der Neuzeit Online, o. D., [http://dx.doi.org/ 10.1163/2352-0248_edn_a179 0000], eingesehen 7.2.2019.

52 Schorlemer, Kulturgutzerstörung, S. 151.

53 Ebd., S. 131-133. 
Dass aber Zerstörungsakte dieser Art ausschließlich aus religiösen Motivationen heraus geschehen, darf natürlich angezweifelt werden. Obwohl sie den Anschein erwecken, spontane Aktionen darzustellen, gehen ihnen meist systematische Planungen voraus, um sie anschließend möglichst öffentlichkeitswirksam verbreiten zu können. Diese öffentliche Inszenierung dient dann nicht nur als Machtdemonstration gegenüber bestimmten Wertesystemen, Staaten(-gemeinschaften) sowie ihren Institutionen, sondern hat auch den Zweck, eine propagandistische Wirkung für die Rekrutierung neuer Mitglieder zu entfalten. Von zunehmender Wichtigkeit ist dabei die Nutzung des Mediums Internet.54 Ebenfalls erwähnt sei - neben der Zerstörung - die Plünderung gewisser Stätten zur Finanzierung der jeweiligen Terrororganisation. Allerdings würde eine detaillierte Beleuchtung all dieser Faktoren den Rahmen der vorliegenden Arbeit bei weitem sprengen, sodass im Folgenden der Fokus auf die rechtlichen Aspekte des Kulturgüterschutzes gelegt werden soll.

\section{3. Über die strafrechtliche Verfolgung von Kulturgutzerstörung}

\subsection{Historischer Abriss}

Während die Plünderung, Beschädigung und Vernichtung von Kulturgütern die gewaltsamen Konflikte der Menschheit seit jeher begleitet haben dürften, begann erst in der zweiten Hälfte des 19. Jahrhunderts ein Verrechtlichungsprozess dieses Bereiches, der zunächst auf der nationalen Ebene einsetzte. Eine der ersten nationalen Verordnungen respektive Empfehlungen dieser Art stellten die 1863 vor dem Hintergrund des Amerikanischen Bürgerkrieges entstandenen Instructions for the Government of Armies of the United States in the Field, auch Lieber Code genannt, dar. In ihnen wurden Plünderungen zwar nicht per se geächtet, jedoch wurden die Streitkräfte der Vereinigten Staaten angewiesen, das Eigentum von Kirchen, Krankenhäusern oder anderen Einrichtungen, die einen ausschließlich gemeinnützigen Charakter aufwiesen, aber auch von Museen und Bildungseinrichtungen jeglicher Art davon auszunehmen. Kunstwerke, Bibliotheken und wissenschaftliche Sammlungen sollten unter allen Umständen vor Schaden behütet werden. Sie durften im Falle einer Entwendung nach dem Artikel 36 des Codes weder verkauft, beschädigt noch zerstört werden. ${ }^{55}$

Wenig später untersagte auch die Brüsseler Deklaration von 1874 die Zerstörung von feindlichem Eigentum, wenn dies aus militärischer Sicht nicht zwingend notwendig sei. Artikel 17 der Deklaration hob auch dezidiert die Schonung von Gebäuden, die der Religion, Kunst, Wissenschaft oder gemeinnützigen Zwecken gewidmet waren, hervor. Sie spiegelt somit einen ersten internationalen Versuch bezüglich des Schutzes von Kulturgut wider, der allerdings nie zum verbindlichen Recht wurde. ${ }^{56}$ Ähnliche Intentionen verfolgten die Haager Abkommen von 1899 und 1907. Ergänzend zu den bisherigen Forderungen legte die Haager Landkriegsordnung fest, dass Gebäude und Monumente von kulturellem, historischem, künstlerischem oder religiösem Wert

54 Schorlemer, Kulturgutzerstörung, S. 151.

55 Drazewska, The Human Dimension, S. 207.

56 Ebd. 
- unter der Voraussetzung, dass sie nicht militärisch genutzt wurden - unangetastet bleiben sollten. Diese Bedingung der militärischen Notwendigkeit als Voraussetzung für den Angriff auf eigentlich geschützte Kulturgüter sollte auch in zukünftigen Abkommen erhalten bleiben. Die mit der Landkriegsordnung verbundenen Hoffnungen wurden allerdings durch die Schrecken des Ersten Weltkrieges zerstreut. ${ }^{57}$ In der Zwischenkriegszeit unternahm der russische Maler, Schriftsteller und Archäologe Nikolai Roerich daher einen erneuten Versuch, das kulturelle Erbe der Menschheit juristisch zu bewahren. Der Entwurf geht dabei im Speziellen auf die unbeweglichen Objekte ein (wie historische Monumente, Museen, wissenschaftliche, kulturelle oder künstlerische Institutionen sowie Bildungsinstitutionen) und legte ebenfalls fest, dass der Schutz bei der militärischen Nutzung der jeweiligen Gebäude verloren ging. Allerdings fand auch der "Roerich-Pakt" außerhalb des amerikanischen Kontinents kaum Beachtung. ${ }^{58}$

Aufmerksamen Leser*innen wird nicht entgangen sein, dass bis zu dieser Stelle von einer strafrechtlichen Verfolgung noch keine Rede war. Die bislang präsentierten Abkommen beinhalteten zwar Vorstellungen, welche Güter und Einrichtungen als schützenswert empfunden wurden, gaben aber keinerlei Auskunft über etwaige Sanktionierungen, die im Falle ihres Bruchs in Kraft treten könnten. Diesbezüglich stellten die Prozesse des Nürnberger Strafgerichtshofes 1945/46 einen ersten bedeutenden Einschnitt dar. Die sich an der Haager Landkriegsordnung von 1907 sowie dem bereits bestehenden Völkergewohnheitsrecht orientierende Charter of the International Military Tribuna/59 legt in ihrem sechsten Artikel die Plünderung von öffentlichen und privaten Besitztümern sowie die militärisch nicht gerechtfertigte Zerstörung von Ortschaften und folglich auch den in ihnen vorhandenen kulturellen Stätten - als Strafbestand fest. Wesentlich waren hierbei zwei Aspekte, welche die Weichen für eine künftige internationale Strafgerichtsbarkeit stellen sollten:

Erstens konnte das Nürnberger Tribunal mit den anschließend durchgeführten Prozessen und Verurteilungen das in den Statuten festgelegte Recht tatsächlich durchsetzen, wodurch die in den letzten Jahrzehnten gewachsenen internationalen Konventionen maßgeblich berücksichtigt wurden. ${ }^{60}$ Gerhard Werle spricht dem Nürnberger Statut in diesem Zusammenhang sogar den Status einer "Geburtsurkunde des Völkerstrafrechts"61 zu. Zweitens hoben die Verhandlungen die individuelle Verantwortlichkeit der Täter*innen hervor. Im Urteil vom 1. Oktober 1946 wird dabei explizit erwähnt, dass dem Völkerrecht nur durch die Bestrafung von einzelnen Personen Geltung verschafft werden kann.62 Obgleich niemand in Nürnberg allein wegen der Zer-

57 Zu den berüchtigtsten Beispielen bewusster Vernichtungsakte wären hier die Zerstörung der im Hochmittelalter erbauten flandrischen Tuchhallen in Ypern, die schwere Beschädigung der Kathedrale von Reims sowie der Verlust der Universitätsbibliothek von Löwen zu nennen: Elena Syssoeva, Kunst im Krieg. Eine völkerrechtliche Betrachtung der deutsch-russischen Kontroverse um kriegsbedingt verbrachte Kulturgüter (Schriften zum Völkerrecht 152), Berlin 2004, S. 246-247.

58 Drazewska, The Human Dimension, S. 208-209.

59 Die im Deutschen geläufige Bezeichnungen wären „Statut des Internationalen Militärgerichtshofs von Nürnberg" bzw., IMG-Statut“: Werle, Völkerstrafrecht, S. 8.

60 Schorlemer, Kulturgutzerstörung, S. 631-632.

61 Werle, Völkerstrafrecht, S. 8.

62 Ebd., S. 9. 
störung von Kulturgütern ${ }^{63}$ auf der Anklagebank saß, lässt sich in dem Verfahren gegen Alfred Rosenberg ein verwandtes Beispiel finden. Er wurde neben zahlreichen anderen Straftaten vom Gericht auch aufgrund der gewaltsamen Plünderung von knapp 22.000 Kunstobjekten als schuldig befunden. ${ }^{64}$

Als weitere Nachwirkung des Zweiten Weltkrieges kam es zur Ausfertigung der Haager Konvention von 1954. Diese war weitaus weniger idealistisch als ihre Vorgängerabkommen und enthielt weiterhin die oben erwähnte „Bedingung der militärischen Notwenigkeit". Allerdings kamen nun zahlreiche Verpflichtungen hinzu, die von den Streitkräften beachtet werden mussten: Die Entwendung oder Requirierung von Kulturgütern wurde untersagt. Ebenso durften sie keinerlei Schäden ausgesetzt werden. Die Unterzeichnerstaaten willigten ein, absichtliche Beschädigungen, die nicht aus militärischen Zwecken notwendig waren, abzuwenden sowie Diebstähle zu unterbinden. Insgesamt ist die Konvention eine pragmatische Lösung für die Militärs und als Minimalkonsens der damaligen Staatengemeinschaft zu deuten. Trotz allem spiegelt sie ein wachsendes Bewusstsein in Bezug auf die Bedeutung von Kulturgütern als gemeinsames Erbe der Menschheit wider. Ihre Präambel beleuchtet diesbezüglich, dass die Vernichtung von Kulturgut - unabhängig von welcher Bevölkerungsgruppe es stammt - einen Schaden für die Gesamtheit aller Menschen nach sich zieht.

Das 1999 verabschiedete zweite Protokoll brachte eine Abschwächung des Grundsatzes der militärischen Notwendigkeit. Ein Grund war neben der fortschreitenden Entwicklung des humanitären Völkerrechts paradoxerweise auch der technologische Fortschritt bei der Entwicklung von Waffen, die zunehmend präziser arbeiteten und damit die Hoffnung weckten, dass Kollateralschäden vermieden werden und Kulturstätten innerhalb von Kampfgebieten verschont bleiben könnten. ${ }^{65}$ Nach dem vierten Artikel des Protokolls dürfen feindselige Handlungen gegen ein Kulturgut nur durchgeführt werden, wenn dieses vorher durch seine Nutzung zu einem militärischen Ziel gemacht wurde und zudem „keine andere durchführbare Möglichkeit besteht, einen ähnlichen militärischen Vorteil zu erlangen, wie er sich bietet, wenn eine feindselige Handlung gegen dieses Ziel gerichtet wird"66. Die endgültige Befehlsgewalt über einen Angriff auf derartige Ziele liegt nach der Konvention bei hochrangigen Offizierspersönlichkeiten. Falls die Entscheidung positiv ausfällt, sollte eine Warnung vorausgeschickt werden. ${ }^{67}$

Es ist an dieser Stelle anzumerken, dass bei den in allen Abkommen angesprochenen militärischen Konflikten die Verfasser*innen von kriegerischen Handlungen zwischen Staaten und ihren nationalen Streitkräften ausgingen - eine Annahme, die nicht mehr

63 Da eine genauere Behandlung der beispiellosen Schäden des Zweiten Weltkriegs den Rahmen dieser Arbeit sprengen würde, sei an dieser Stelle auf andere, zahlreich vorhandene Einzelstudien, welche die Vernichtung und den Raub von Kulturgütern durch alle Kriegsparteien thematisieren, verwiesen. Als Beispiel wäre hier die Monografie von Elena Syssoeva zu nennen: Syssoeva, Kunst im Krieg.

64 Schorlemer, Kulturgutzerstörung, S. 631-633.

65 Drazewska, The Human Dimension, S. 209-210.

66 Rechtsinformationssystem des Bundes (Hrsg.), Gesamte Rechtsvorschrift für Konvention zum Schutz von Kulturgut bei bewaffneten Konflikten. 2. Protokoll, 26.3.1999, [https://www.ris. bka.gv.at/GeltendeFassung.wxe?A bfrage $=$ Bundesnormen\&Gesetzesnummer $=20003628$ ], eingesehen 9.3.2019. 
auf die Zustände der jüngeren Vergangenheit zutrifft. Die sich während den vergangenen Jahrzehnten entwickelnde asymmetrische Kriegsführung, bei der (inter-)nationale Truppen meist nichtstaatlichen Terror- oder Rebellenorganisationen gegenüberstehen, schuf neue Rahmenbedingungen und Herausforderungen für die juristische Durchsetzung des Kulturgüterschutzes. Hinzu kommt, dass sich diese nichtstaatlichen Gruppierungen neuer Zerstörungsmuster bedienen, die sich von früheren bewaffneten Konflikten unterscheiden, auf welche die vorgestellten Konventionen ausgelegt sind. Gerade die vieldiskutierten Regelungen zur militärischen Notwendigkeit von Zerstörungen werden hierbei untergraben, denn die Angriffe von Gruppen (wie Ansar Dine oder dem „Islamischen Staat") gegen Kulturgüter hatten nie den Zweck, einen militärischen Vorteil zu erringen. Sie fanden vielmehr nach den Kampfhandlungen und während der Okkupation der betroffenen Gebiete statt. Klassische militärische Rechtfertigungen - wie die Betrachtung eines zerstörten Objekts als Kollateralschaden spielen keine Rolle mehr. ${ }^{68}$

Als ein erster Versuch, auf diese Entwicklungen einzugehen, kann die UNESCO Declaration concerning the Intentional Destruction of Cultural Heritage vom Oktober 2003 gesehen werden. Sie stellt eine direkte Reaktion der UNESCO auf die Vernichtung der Buddhas von Bamiyan durch die Taliban dar. Ihr Inhalt thematisiert - ganz allgemein - die absichtliche Zerstörung von Kulturgütern auf nationaler sowie internationaler Ebene während militärischer Konflikte sowie in Perioden des Friedens und unabhängig davon, ob die betroffenen Objekte in einem speziellen Register aufgelistet sind, wie es unter anderem das zweite Protokoll von 1999 vorsieht. ${ }^{69}$ Dabei wird in Artikel VII nicht nur die Verantwortlichkeit der einzelnen Staaten, sondern auch die strafrechtliche Verfolgung von Individuen betont. Explizit fordert die UNESCO die Etablierung einer dementsprechenden Gerichtsbarkeit auf der Grundlage des bestehenden internationalen Rechts.

„States should take all appropriate measures, in accordance with international law, to establish jurisdiction over, and provide effective criminal sanctions against, those persons who commit, or order to be committed, acts of intentional destruction of cultural heritage of great importance for humanity, whether or not it is inscribed on a list maintained by UNESCO or another international organization." ${ }^{\prime 70}$

Es scheint also, dass die juristische Einforderung dieser individuellen Verantwortlichkeit als ein Schlüsselbaustein zur effizienteren Bekämpfung der jüngeren Angriffe gegen Kulturgüter wahrgenommen wurde. Ihre historische Entwicklung und konkrete Umsetzung im Prozess gegen Ahmad Al Mahdi wird nun genauer beleuchtet.

68 Schorlemer, Kulturgutzerstörung, S. 150

69 Drazewska, The Human Dimension, S. 211

70 United Nations Educational, Scientific and Cultural Organization (Hrsg.), UNESCO Declaration concerning the Intentional Destruction of Cultural Heritage, 17.10.2003, [http://portal.unesco. org/en/ev.php-URL_ ID=17718\&URL_DO=DO_TOPIC\&URL_SECTION=201.html], eingesehen 12.3.2019. 


\subsection{Entwicklungen seit 1990 und die Einrichtung des Internationalen Strafgerichtshofs}

Trotz der Grundsteinlegung für eine internationale Strafgerichtsbarkeit 1946 schienen die Nürnberger Prozesse vorerst nur ein Zwischenspiel zu sein. Erneut folgte die Verabschiedung von Konventionen, deren Missachtung keine klar geregelte strafrechtliche Verfolgung nach sich zog. Die Spannungen des Kalten Krieges erschufen diesbezüglich eine zwiespältige Situation, in der zwar die Grundlagen des Völkerstrafrechts größtenteils gesichert waren, eine konsequente Anwendung aber scheiterte. Erst der Fall des Eisernen Vorhangs brachte hier eine erneute Dynamik ein. Konkrete Anlässe zur Reaktivierung der in Nürnberg erprobten Praxis ergaben sich durch die schweren Verletzungen des humanitären Völkerrechts im Jugoslawienkrieg sowie durch den Völkermord in Ruanda. Die Stellung, welche die Alliierten in Nürnberg eingenommen hatten, wurde nun durch die Vereinten Nationen (UN) vertreten. Auf Grundlage des Kapitels VII der UN-Charta, das Maßnahmen bezüglich der Wahrung respektive der Wiederherstellung des internationalen Friedens zum Inhalt hat, schuf der UN-Sicherheitsrat durch eine Resolution zwei internationale Strafgerichtshöfe. In der Literatur werden sie aufgrund dieses Vorgehens als „Ad-hoc-Strafgerichtshöfe“ bezeichnet. Ebenfalls zwingt die Einrichtung der Strafgerichtshöfe durch eine Resolution jeden Staat der UN zur Zusammenarbeit mit diesen und räumt ihnen eine vorrangige Zuständigkeit - auch gegenüber den nationalen Gerichtshöfen - ein. ${ }^{71}$

Von besonderer Bedeutung für die Ausführungen dieser Arbeit ist das Statut für den Jugoslawien-Strafgerichtshof. Dieses Statute of the International Criminal Tribunal for the Former Yugoslavia oder ICTY-Statut, ${ }^{72}$ das der Sicherheitsrat durch die Resolution 827 vom 27. Mai 1993 verabschiedete, bezieht sich vor allem in seinem dritten Artikel auf die strafrechtliche Ahndung von Verbrechen gegen Kulturgüter. Erstmals können einzelne Personen nicht nur wegen der Inbesitznahme oder der Beschädigung dieser, sondern auch wegen ihrer Zerstörung sanktioniert werden. ${ }^{73}$

"The International Tribunal shall have the power to prosecute persons violating the laws or customs of war. Such violations shall include, but not be limited to: [...] d) seizure of, destruction [sic!] or wilful damage done to institutions dedicated to religion, charity and education, the arts and sciences, historic monuments and works of art and science"."74

Unter diesen Eindrücken verstetigte sich immer mehr der Gedanke, einen ständigen internationalen Strafgerichtshof einzurichten. Bereits vor dem Zweiten Weltkrieg gab es erste Versuche dieser Art, die aber letztendlich scheiterten. ${ }^{75}$ Nun war mit der Neu-

71 Werle, Völkerstrafrecht, S. 18-19.

72 International Tribunal for the Prosecution of Persons Responsible for Serious Violations of International Humanitarian Law Committed in the Territory of the Former Yugoslavia since 1991 (Hrsg.), Updated Statute of the International Criminal Tribunal for the Former Yugoslavia, September 2009, [http://www.icty.org/x/file/Legal\%20 Library/Statute/statute_sept09_en.pdf], eingesehen 17.3.2019.

73 Schorlemer, Kulturgutzerstörung, S. 642.

74 International Tribunal for the Prosecution of Persons Responsible for Serious Violations of International Humanitarian Law Committed in the Territory of the Former Yugoslavia since 1991, Updated Statute, S. 5.

75 Werle, Völkerstrafrecht, S. 23-24. 
ordnung der geopolitischen Verhältnisse in den 1990er-Jahren ein günstiger Zeitpunkt gekommen, um ein solches Vorhaben tatsächlich umzusetzen. Auf Grundlage der Ad-hoc-Gerichtshöfe konnte die Völkerrechtskommission der Vereinten Nationen bereits 1994 einen ersten Entwurf für ein Statut eines internationalen Strafgerichtshofes präsentieren. Nach einer weiteren Überarbeitungsperiode tagte ab dem 16. Juni 1998 eine von den Vereinten Nationen in Rom einberufene internationale Konferenz, bestehend aus Vertreter*innen von Staaten, zwischenstaatlichen Organisationen sowie Nichtregierungsorganisationen. Nach Werle standen sich von Beginn an ein gerichtshoffreundliches sowie ein skeptisches Lager gegenüber. Letzterem gehörten Staaten an, die aus Sorge um ihre nationale Souveränität keinen ständigen Gerichtshof wünschten, ${ }^{76}$ sondern eine Art Ad-hoc-Gerichtshof, der aber nur vom Sicherheitsrat der Vereinten Nationen in konkreten Krisensituationen aktiviert werden sollte. ${ }^{77}$

Letztendlich konnte sich die Mehrheit der Konferenzteilnehmer*innen auf einen Entwurf einigen und nahm das Rome Statute of the International Criminal Court am 17. Juli 1998 mit 120 Stimmen an. Sieben Staaten, nämlich die USA, China, Israel, Irak, Libyen, Jemen sowie Katar lehnten das Statut ab, während sich 21 Staaten der Abstimmung enthielten. Als Voraussetzung für das Inkrafttreten des Statuts mussten über sechzig Nationen das Dokument ratifizieren, was im April 2002 der Fall war, sodass das IStGHStatut mit dem 1. Juli 2002 wirksam wurde. Am 11. März 2003 nahm der Internationale Strafgerichtshof, mit Sitz in Den Haag, seine Arbeit auf. ${ }^{78}$ Im März 2019 hatte das Statut 122 Vertragsstaaten, wobei die Mehrheit der ständigen Mitglieder des UN-Sicherheitsrates (China, Russland und die USA) nicht hinzuzählte. ${ }^{79}$

Was die Funktionsweise des IStGH betrifft, so ist zu sagen, dass dieser komplementär zur Gerichtsbarkeit seiner Mitgliedsstaaten arbeitet. Nach seinem Statut kann er immer dann eingreifen, wenn es einem Staat nicht gelingen sollte, seiner strafrechtlichen Verantwortung nachzukommen. Zur Einleitung eines Verfahrens gibt es zwei Möglichkeiten: Entweder wendet sich ein Mitgliedsstaat des Statuts von Rom an die Anklagebehörde des Gerichtshofes oder ein*e Ankläger*in beginnt selbstständig mit den Ermittlungen. Falls aber ein Staat das IStGH-Statut noch nicht unterzeichnet respektive ratifiziert hat, besteht auch die Möglichkeit der Überweisung von Straffällen durch den Sicherheitsrat der Vereinten Nationen. Dies war bereits bei Libyen der Fall. Verbrechen, die sich im Zuge des Syrien-Konflikts ereigneten, kamen bisher durch die innere Uneinigkeit des Sicherheitsrates in dieser Frage noch nicht zur Anklage. ${ }^{80}$ Ansonsten könnte sich auch hier ein ähnlicher Prozess wie der von Ahmad Al Mahdi ergeben.

Inwiefern kann nun der IStGH bei Verbrechen gegen Kulturgüter eingreifen? Zunächst kann ein rechtlicher Schutz aus dem Artikel 8 des Römischen Statuts, welcher den Tat-

\footnotetext{
76 Werle nennt hier beispielsweise die Vereinigten Staaten, Indien und China: ebd., S. 25.

77 Ebd.

78 Ebd., S. 27-28.

79 International Criminal Court - Secretariat of the Assembly of States Parties (Hrsg.), The States Parties to the Rome Statute, o. D., [https://asp.icc-cpi.int/en_menus/asp/states\%20parties/pages/the \%20states\%20parties\%20 to\%20the\%20rome\%20statute.aspx], eingesehen 18.3.2019. 
bestand der Kriegsverbrechen definiert, abgleitet werden. Dieser behandelt zwar nicht explizit Angriffe gegen Kulturgüter, ächtet jedoch die Zerstörung, Plünderung oder Requirierung von zivilem Eigentum. Dabei ist anzumerken, dass die beiden letzten Maßnahmen erneut von der militärischen Notwendigkeit abhängig sind. Etwas konkreter sind hingegen die ebenfalls in Artikel 8 dargelegten Strafbestände, die sich an der Haager Landkriegsordnung orientieren, aber nun auch erstmals Angriffe gegen unbewegliche geschützte Objekte innerhalb von nicht-internationalen bewaffneten Konflikten als Kriegsverbrechen bewerten. ${ }^{81}$ Dazu zählen "attacks against buildings dedicated to religion, education, art, science or charitable purposes, historic monuments, hospitals and places where the sick and wounded are collected, provided they are not military objectives" 82 .

Entscheidend für die Verurteilung Al Mahdis ist neben diesen Definitionen der bereits angesprochene Aspekt der individuellen strafrechtlichen Verantwortlichkeit, der in Artikel 25 des IStGH-Statuts formuliert ist. Sabine von Schorlemer erwähnt diesen Artikel in ihrer - ansonsten sehr ausführlichen - Monografie nicht, da diese wohl vor dem endgültigen Urteilsspruch im September $2016^{83}$ erschien. Al Mahdi machte sich demnach in insgesamt vier Punkten, die sich alle auf die Gesamtheit der im Statut definierten Straftaten beziehen, nach Artikel 25 strafbar.

"[A] person shall be criminally responsible and liable for punishment for a crime within the jurisdiction of the Court if that person: (a) Commits such a crime, whether as an individual, jointly with another or through another person, regardless of whether that other person is criminally responsible;

(b) Orders, solicits or induces the commission of such a crime which in fact occurs or is attempted;

(c) For the purpose of facilitating the commission of such a crime, aids, abets or otherwise assists in its commission or its attempted commission, including providing the means for its commission; (d) In any other way contributes to the commission or attempted commission of such a crime by a group of persons acting with a common purpose.".84

\section{The Prosecutor v. Ahmad Al Faqi Al Mahdi}

\subsection{Ermittlungen, Inhaftierung und Prozess}

Nachdem nun die rechtlichen Grundlagen für die Verurteilung Al Mahdis erläutert wurden, soll abschließend die Person des Verurteilten, der Ablauf des Prozesses sowie dessen Rezeption genauer beleuchtet werden. Ahmad Al Faqi Al Mahdi wurde laut

81 Schorlemer, Kulturgutzerstörung, S. 644-646.

82 International Criminal Court (Hrsg.), Rome Statute of the International Criminal Court, 16.1.2002, S. 9, [https:// www.icc-cpi.int/nr/rdonlyres/ea9aeff7-5752-4f84-be94-0a655eb30e16/0/rome_statute_english.pdf], eingesehen 19.3.2019.

83 International Criminal Court, Case Information Sheet.

84 International Criminal Court, Rome Statute, S. 17. 
eigenen Angaben in einem Dorf in der Umgebung von Timbuktu geboren und sieht sich als Angehöriger der Tuareg. Zum Zeitpunkt der Anklage 2016 dürfte er in etwa vierzig Jahre alt gewesen sein. Nach der Einnahme von Timbuktu war Al Mahdi als Kopf der sogenannten Hesba, einer der Ansar Dine zugehörigen Sittenpolizei, tätig. ${ }^{85}$ Diese Funktion übte Al Mahdi ab dem Zeitpunkt seiner Ankunft in Timbuktu im April 2012 bis zum September desselben Jahres aus, wobei er als Experte in religiösen Fragen konsultiert wurde. Im Auftrag von Ansar Dine überwachte er die Mausoleen und Ruhestätten der Stadt und setzte das Verbot der bisherigen religiösen Praktiken um. Ende Juni 2012 entschied sich die Führung der Gruppe schließlich zur Zerstörung der Mausoleen, was sie an Al Mahdi, der dies umsetzen sollte, weiterleiteten. Nach anfänglichen Einwänden folgte Ahmad Al Mahdi schließlich diesen Instruktionen und machte sich nach den oben aufgezählten Tatbeständen strafbar. Er sorgte nicht nur für die Heranschaffung der notwendigen Werkzeuge und bereitete logistische Maßnahmen vor, sondern legte über dies hinaus die Reihenfolge der Aktionen fest, beaufsichtigte deren Ausführung und nahm an der Zerstörung der Mausoleen in mindestens fünf Fällen teil. Außerdem rechtfertigte er die zwischen dem 30. Juni und 11. Juli 2012 erfolgten Angriffe vor der Öffentlichkeit in einer vorausgehenden Predigt sowie vor Journalist*innen. ${ }^{86}$

Die Eröffnung der (Vor-)Untersuchungen geschah auf Initiative der malischen Regierung, die sich am 13. Juli 2012 an den Internationalen Strafgerichtshof wandte. ${ }^{87} \mathrm{Im}$ Januar 2013 verkündete die Chefanklägerin des IStGH, Fatou Bensouda, das Eingreifen des Gerichtshofes, da sie in den Taten Kriegsverbrechen nach Artikel 8 des Römischen Statuts sah. ${ }^{88}$ Daraufhin begannen am 13. Januar 2013 die offiziellen Ermittlungen. Am 18. September 2015 erging aufgrund des Kriegsverbrechens der absichtlichen Angriffe gegen historische Monumente sowie Gebäude, welche der Religionsausübung gewidmet sind, ein Haftbefehl gegen Al Mahdi. Die Auslieferung des Angeklagten, der sich zu diesem Zeitpunkt in Gewahrsam von nigerianischen Sicherheitskräften befand, erfolgte bereits am 26. September. Nach dem darauffolgenden Transfer in die Niederlande wurde Al Mahdi am 30. September erstmals den Richter*innen vorgeführt und über seine Rechte in einer Sprache informiert, welche er einwandfrei beherrscht und spricht - in diesem Fall Arabisch. Ein knappes Jahr darauf fand der Prozess innerhalb weniger Tage, vom 22. bis 24. August 2016, statt. Außer dem Angeklagten selbst wurden noch neun weitere Zeug*innen respektive Opfer angehört. Das Gericht befand Ahmad Al Faqi Al Mahdi am 27. Dezember 2016 gemäß Artikel 25 (3) (a) bis (d) des IStGH-Statuts für schuldig und verurteilte ihn neben einer neunjährigen Freiheitsstrafe zu Reparationszahlungen in Höhe von 2,7 Millionen Euro. ${ }^{89}$

85 Charlotte Louise Joy, Crimes against Cultural Heritage in Timbuktu, in: Anthropology Today 34 (2018), Heft 1, S. 1517, hier S. 15.

86 Uzma S. Bishop-Burney, Prosecutor v. Ahmad Al Faqi Al Mahdi, in: American Journal of International Law 111 (2017), Heft 1, S. 126-132, hier S. 16-17.

87 International Criminal Court, Case Information Sheet.

88 Schorlemer, Kulturgutzerstörung, S. 662.

89 International Criminal Court, Case Information Sheet. 


\subsection{Bewertung des Prozesses}

Neben der Kürze des Verfahrens sind noch zwei weitere Sachverhalte bemerkenswert: Ahmad Al Mahdi war nicht nur der erste Angeklagte, der wegen der Zerstörung von Kulturgut vor dem IStGH angeklagt wurde, sondern auch der erste, welcher auch ein Geständnis bezüglich seiner Taten ablegte..$^{90}$ Dieses stellte eines von insgesamt fünf mildernden Umständen dar (neben dem anfänglichen Widerstreben zur Durchführung der Straftat, seinem guten Verhalten während der Haft, der Kooperation mit dem Tribunal sowie der von ihm an den Tag gelegten Reue und Empathie für die Opfer), aus denen sich die Höhe des Urteils ergab. ${ }^{91}$ Beobachter ${ }^{*}$ innen hegten diesbezüglich auch die Hoffnung, dass sich das Geständnis Al Mahdis positiv auf die laufenden Verfahren gegen andere Kriegsverbrechen in Mali seit $2012^{92}$ auswirken könnte. ${ }^{93}$

Im Vergleich zur Rechtsprechung des Internationalen Strafgerichtshofes für das ehemalige Jugoslawien, welche zwar die Zerstörung und vorsätzliche Beschädigung von Kulturgütern, nicht aber die Angriffe auf diese berücksichtigte, schaffte das Urteil des IStGH den Rahmen für die weitere Entwicklung des rechtlichen Kulturgüterschutzes innerhalb bewaffneter Konflikte. Auch ist zu erwähnen, dass es sich bei den in Jugoslawien zerstörten Objekten - wie die Brücke von Mostar, die diesen Status erst 2005 erhielt - nicht ausschließlich um Weltkulturerbestätten handelte. Die Mausoleen von Timbuktu waren zwar als solche von der UNESCO aufgelistet, was jedoch in Bezug auf Artikel acht des IStGH-Statutes keine Rolle spielte. Letztendlich bestätigte das Urteil gegen Al Mahdi also den allgemeinen Wert von Kulturgütern - unabhängig von der Bewertung durch die UNESCO. Allerdings ist zu erwähnen, dass die UNESCO-Expertise - auch wenn diese formal-rechtlich keine Relevanz besitzt - als Indikator dient, welche Güter als schützenswert gelten. Diese Grauzone bleibt also auch nach dem Fall Al Mahdis erhalten. ${ }^{94}$

Was die Bedeutung des Prozesses als Präzedenzfall betrifft, existieren gegensätzliche Meinungen. Einerseits erhoben skeptische Stimmen den Vorwurf, dass die Schwere der Straftaten Al Mahdis nach der Definition des Römischen Statuts nicht ausreichen würde, um überhaupt einen Prozess auf internationaler Ebene zu rechtfertigen. ${ }^{95}$ Das Festhalten an solchen Positionen würde weitere Prozesse dieser Art erschweren. Andererseits könnte das Al Mahdi Urteil als Ausgangsbasis für vergleichbare Verfahren, betreffend der Straftaten gegen das kulturelle Erbe Syriens, Afghanistans und des Iraks, dienen. ${ }^{96}$

90 Joy, Crimes, S. 15.

91 Bishop-Burney, Prosecutor, S. 129

92 International Criminal Court (Hrsg.), Mali, o. D., [https://www.icc-cpi.int/mali], eingesehen 23.3.2019.

93 Milena Sterio, Individual Criminal Responsibility for the Destruction of Religious and Historic Buildings. The Al Mahdi Case, in: Case Western Reserve Journal of International Law 49 (2017), Heft 1, S. 63-73, hier S. 68.

94 Bishop-Burney, Prosecutor v. Ahmad, S. 130-132.

95 Sterio, Individual Criminal Responsibility, S. 70-73.

96 Juan-Pablo Pérez-León-Acevedo, International Criminal Justice Rendered Concerning the Attack Against Timbuktu Mausoleums and Mosque. Focus on Religion-Related Considerations, in: Oxford Journal of Law and Religion 6 (2017), Heft 1, S. 180-186, hier S. 186. 


\section{Fazit}

Wie die vorliegenden Ausführungen zeigten, konnte der juristische Kulturgüterschutz über die letzten 150 Jahre stetig weiterentwickelt werden. Während die vor 1945 ausgearbeiteten Entwürfe zwar grundlegende Ideen einbrachten, fanden sie in konkreten Kriegssituationen aufgrund ihrer fehlenden strafrechtlichen Konsequenzen nur wenig Beachtung. Dennoch spielten sie für die Folgeabkommen, denen sie als Rechtsgrundlagen dienten, eine tragende Rolle. Wegweisende Einschnitte brachten die Erfahrungen des Zweiten Weltkriegs, in dessen Folge die transnationale Zusammenarbeit ausgebaut wurde. Die daraus resultierenden Nürnberger Prozesse leisteten mit einer ersten Definition von Kriegsverbrechen sowie dem Prinzip der individuellen strafrechtlichen Verantwortlichkeit einen essentiellen Beitrag zur Arbeitsweise der Ad-hoc Gerichte der 1990er und des aus ihnen entstandenen Internationalen Strafgerichtshofes. Gerade die Fokussierung auf Einzelpersonen ist eine wichtige Voraussetzung, um strafrechtlich gegen nichtstaatliche Streitkräfte respektive Terrororganisationen vorgehen zu können. Vor allem der Artikel 25 des IStGHs, auf dessen Grundlage Ahmad Al Mahdi verurteilt wurde, stellt in dieser Hinsicht ein wertvolles Werkzeug dar.

Die Frage, ob die Verurteilung Al Mahdis letztendlich einen nachhaltigen Einschnitt in die strafrechtliche Ahndung der Zerstörung von Kulturgütern bringt, kann nicht vollends beantwortet werden, da durch den kurzen zeitlichen Abstand noch kein ähnlicher Fall am IStGH verhandelt wurde. Allerdings gehen aus den dargelegten Ausführungen einige Argumente für die Bedeutung des Prozesses hervor. So stellt das Zustandekommen eines solchen Verfahrens eine gewichtige symbolische Geste dar und spiegelt ein wachsendes Bewusstsein für die Bedeutung des kulturellen Erbes der Menschheit wider. Das vom Gericht als glaubwürdig eingeschätzte Schuldeingeständnis von Al Mahdi stützt diese Feststellung zusätzlich. Des Weiteren verschärfte das Urteil des IStGH die allgemeine Gangart gegen Verbrechen an Kulturgütern. Erstmals wurde nicht nur die Beschädigung oder Zerstörung, sondern bereits der Angriff auf Kulturgüter als Straftat gewertet. Drei der insgesamt vier Anklagepunkte bezogen sich nicht allein auf die Durchführung der Zerstörungen, sondern auch auf deren Unterstützung und die Aufforderung zu diesem Verbrechen.

Schlussendlich ergibt sich das Bild, dass nur ein internationaler Gerichtshof der strafrechtlichen Verfolgung von modernen Terrororganisationen gewachsen zu sein scheint, wobei die Anklage gegen Al Mahdi einen Vorbildcharakter entwickeln könnte. Mit der Unterstützung des UN-Sicherheitsrates - vorausgesetzt er erteilt diese - wäre es dem IStGH möglich, angemessene Maßnahmen gegenüber den jüngsten Verbrechen an Kulturgütern im Nahen Osten zu ergreifen.

\section{Literatur und Quellen}

Afsaruddin, Asma, ulamā, in: Encyclopædia Britannica, 27.3.2018, [https://www. britannica.com/topic/ulama], eingesehen 16.2.2019. 
Ansar Dine Fighters Destroy Timbuktu Shrines, in: Al Jazeera English 1.7.2012, [https://www.aljazeera.com/news/africa/2012/06/2012630101748795606.html], eingesehen 26.2.2019.

Bishop-Burney, Uzma S., Prosecutor v. Ahmad Al Faqi Al Mahdi, in: American Journal of International Law 111 (2017), Heft 1, S. 126-132.

Brüne, Stefan, Problemregion Mali Nord - ein entwicklungspolitischer Rückblick, in: Sicherheit und Frieden 32 (2014), Heft 2, S. 86-90.

Campion, Kristy, Blast through the Past. Terrorist Attacks on Art and Antiquities as a Reconquest of the Modern Jihadi Identity, in: Perspectives on Terrorism 11 (2017), Heft 1, S. 26-39.

Drazewska, Berenika, The Human Dimension of the Protection of the Cultural Heritage from Destruction during Armed Conflicts, in: International Journal of Cultural Property 22 (2015), Heft 2-3, S. 205-228.

Ehrhardt, Christoph/Gutschker, Thomas, Libyen. Loyal zum Stamm, nicht zum Regime, in: Frankfurter Allgemeine Zeitung, 23.2.2011, [https://www.faz.net/-gq9-y4l8], eingesehen 17.2.2019.

International Council on Monuments and Sites (Hrsg.), Advisory Body Evaluation, The Old Town of Timbuktu, No. 119 Rev., 22.12.1987, [https://whc.unesco. org/document/152016], eingesehen 5.1.2019.

International Criminal Court - Secretariat of the Assembly of States Parties (Hrsg.), The States Parties to the Rome Statute, o. D., [https://asp.icc-cpi.int/ en_menus/asp/states\% 20 parties/pages/the\%20states\%20parties\%20to\%20the\% 20rome\%20statute. aspx], eingesehen 18.3.2019.

International Criminal Court (Hrsg.), Case Information Sheet. The Prosecutor v. Ahmad Al Faqi Al Mahdi, 20.3.2018, [https://www.icc-cpi.int/Caselnformation Sheets/Al-MahdiEng.pdf], eingesehen 5.1.2019.

International Criminal Court (Hrsg.), Mali, o. D., [https://www.icc-cpi.int/mali], eingesehen 23.3.2019.

International Criminal Court (Hrsg.), Rome Statute of the International Criminal Court, 16.1.2002, [https://www.icc-cpi.int/nr/rdonlyres/ea9aeff7-5752-4f84-be94-0a655eb30e16/0/rome_statute_english.pdf], eingesehen 19.3.2019.

International Criminal Court (Hrsg.), Statement of the Prosecutor of the International Criminal Court, Fatou Bensouda, at the Opening of the Confirmation of Charges Hearing in the Case against Mr Ahmad Al-Faqi Al Mahdi, 1.3.2016, [https://www.icc-cpi.int/ Pages/item.aspx?name=otp-stat-01-03-16], eingesehen 20.2.2019.

International Tribunal for the Prosecution of Persons Responsible for Serious Violations of International Humanitarian Law Committed in the Territory of the Former Yugoslavia since 1991 (Hrsg.), Updated Statute of the International Criminal Tribunal for the For- 
mer Yugoslavia, September 2009, [http://www.icty.org/x/file/ Legal\%20Library/Statute/statute_sept09_en.pdf], eingesehen 17.3.2019.

Joy, Charlotte Louise, Crimes against Cultural Heritage in Timbuktu, in: Anthropology Today 34 (2018), Heft 1, S. 15-17.

Krings, Thomas, Die Tradition der urbanen Lehmarchitektur im Obernigergebiet von Mali. Kulturgeographische Beobachtungen zum "sudanischen Baustil" in der Stadt Djenné (Mali), in: Die Erde 115 (1984), Heft 1, S. 123-144.

Ders., Sahelländer. Mauretanien, Senegal, Gambia, Mali, Burkina Faso, Niger, Darmstadt 2006.

Lerch, Wolfgang Günter, Zerstörungen in Mali. Steinzeit-Islam, in: Frankfurter Allgemeine Zeitung, 2.7.2012, [https://www.faz.net/aktuell/politik/ausland/zerstoerungen-in-malisteinzeit-islam-11807372.html], eingesehen 23.2.2019.

Österreichische UNESCO-Kommission (Hrsg.), Die Konvention. Übereinkommen zum Schutz des Kultur- und Naturerbes der Welt, o. D., [https://www.unesco.at/kultur/welterbe/die-konvention/], eingesehen 16.2.2019.

Pérez-León-Acevedo, Juan-Pablo, International Criminal Justice Rendered Concerning the Attack Against Timbuktu Mausoleums and Mosque. Focus on Religion-Related Considerations, in: Oxford Journal of Law and Religion 6 (2017), Heft 1, S. 180-186.

Rechtsinformationssystem des Bundes (Hrsg.), Gesamte Rechtsvorschrift für Konvention zum Schutz von Kulturgut bei bewaffneten Konflikten. 2. Protokoll, 26.3.1999, [https://www.ris.bka.gv.at/GeltendeFassung.wxe?Abfrage=Bundesnormen\&Gesetzesnum mer=20003628], eingesehen 9.3.2019.

Reichmuth, Stefan, Scharia, in: Enzyklopädie der Neuzeit Online, o. D., [https://referenceworks.brillonline.com/entries/enzyklopaedie-der-neuzeit/scharia-a3747000?s. num=0\&s.f.s2_parent=s.f.book.enzyklopaedie-der-neuzeit\&s.q=sharia], eingesehen 17.3.2019

Scheen, Thomas, Al Qaida beteiligt? Tuareg richten Massaker im Norden Malis an, in: Frankfurter Allgemeine Zeitung, 14.2.2012, [https://www.faz.net/-gq5-6xose], eingesehen 18.2.2019.

Schorlemer, Sabine von, Kulturgutzerstörung. Die Auslöschung von Kulturerbe in Krisenländern als Herausforderung für die Vereinten Nationen (The United Nations and Global Change 11), Baden-Baden 2016.

Stanford University (Hrsg.), Ansar Dine. Mapping Militant Organizations, 8.8.2016, [http:// web.stanford.edu/group/mappingmilitants/cgi-bin/groups/view/437\#note25], eingesehen 21.2.2019.

Sterio, Milena, Individual Criminal Responsibility for the Destruction of Religious and Historic Buildings: The Al Mahdi Case, in: Case Western Reserve Journal of International Law 49 (2017), Heft 1, S. 63-73. 
Syssoeva, Elena, Kunst im Krieg. Eine völkerrechtliche Betrachtung der deutsch-russischen Kontroverse um kriegsbedingt verbrachte Kulturgüter (Schriften zum Völkerrecht 152), Berlin 2004.

Tetzlaff, Rainer, Afrika. Eine Einführung in Geschichte, Politik und Gesellschaft, Wiesbaden 2018.

Timbuktu Shrines Damaged by Mali Ansar Dine Islamists, in: BBC News, 30.6.2012, [https://www.bbc.com/news/world-africa-18657463], eingesehen 19.2.2019.

Timbuktu's Sidi Yahia Mosque 'Attacked by Mali Militants', in: BBC News, 2.7.2012, [https://www.bbc.com/news/world-africa-18675539], eingesehen 27.2.2019.

United Nations Educational, Scientific and Cultural Organization (Hrsg.), Director-General of UNESCO Calls for a Halt to Destruction of Cultural Heritage Site in Timbuktu, 30.6.2012, [https://whc.unesco.org/en/news/901/], eingesehen 26.2.2019.

United Nations Educational, Scientific and Cultural Organization (Hrsg.), The Criteria for Selection, o. D., [https://whc.unesco.org/en/criteria/], eingesehen 16.2.2019.

United Nations Educational, Scientific and Cultural Organization (Hrsg.), Timbuktu, o. D., [https:// whc.unesco.org/en/list/119/, eingesehen 5.1.2019.

United Nations Educational, Scientific and Cultural Organization (Hrsg.), UNESCO Declaration Concerning the Intentional Destruction of Cultural Heritage, 17.10.2003, [http://portal.unesco.org/en/ev.php-URL_ID=17718\&URL_DO =DO_TOPIC\&URL_SEC$\mathrm{TION}=201 . \mathrm{html}$, eingesehen 12.3.2019.

United Nations Educational, Scientific and Cultural Organization (Hrsg.), UNESCO Director-General Expresses Concern about the Situation in Mali, 2.4.2012, [https://whc. unesco.org/en/news/865/], eingesehen 26.2.2019.

Werle, Gerhard, Völkerstrafrecht, Tübingen $2012^{3}$.

Wiggermann, Uta, Ikonoklasmus, in: Enzyklopädie der Neuzeit Online, o. D., [http:// dx.doi.org/10.1163/2352-0248_edn_a1790000], eingesehen 7.2.2019.

Maximilian Gröber studiert Geschichte sowie Katholische Fachtheologie an der Universität Innsbruck. maximilian.groeber@student.uibk.ac.at

\section{Zitation dieses Beitrages}

Maximilian Gröber, Von militärischer Notwendigkeit zu individueller Verantwortlichkeit - Der Fall "Al Mahdi“ als Zäsur in der strafrechtlichen Ahndung von Kulturgutzerstörung auf internationaler Ebene, in: historia.scribere 12 (2020), S. 83-103, [http://historia. scribere.at], eingesehen 15.6.2020 (=aktuelles Datum).

Creative Commons Licences 3.0 Österreich unter Wahrung der Urheberrechte der Autorlnnen. 
